\title{
Abordagem Desplugada para o Estímulo do Pensamento Computacional de Estudantes do Ensino Fundamental com Histórias em Quadrinhos
}

\author{
Cicero G. Santos ${ }^{1}$, Maria Augusta S. N. Nunes ${ }^{12}$ \\ ${ }^{1}$ Programa de Pós-Graduação em Ciência da Computação - Universidade Federal de \\ Sergipe (UFS) \\ Av. Marechal Rondon, s/n - São Cristóvão - SE - Brasil \\ ${ }^{2}$ Departamento de Informática Aplicada - Universidade Federal do Estado do Rio de \\ Janeiro (UNIRIO) \\ Av. Pasteur, 296 - Urca, Rio de Janeiro - RJ - Brasil \\ \{cic.goncalves.santos, gutanunes\}@gmail.com
}

\begin{abstract}
Observed the difficulties of the students in the subjects of Portuguese Language (textual interpretation) and Mathematics (logical reasoning). This article presents a playful and dynamic approach in the teaching-learning process for the development of Computational Thinking, combining the concepts of Computer Science, Unplugged Activities and Comic Books. The influence of this approach was evaluated with $9^{\text {th }}$-grade students, during 9 weekly meetings of 50 minutes (each). The results showed a better performance of the students in the mentioned disciplines, after the intervention of the Unplugged with the comics approach.
\end{abstract}

Resumo. Observada as dificuldades dos alunos nas disciplinas de Língua Portuguesa (interpretação textual) e Matemática (raciocínio lógico). Este artigo apresenta uma abordagem lúdica e dinâmica no processo de ensinoaprendizagem para o desenvolvimento do Pensamento Computacional, combinando os conceitos de Ciência da Computação, atividades Desplugadas e Histórias em Quadrinhos. Avaliou-se a influência desta abordagem com alunos do $9^{\circ}$ ano do ensino fundamental, durante 9 encontros semanais de 50 minutos (cada). Os resultados evidenciaram melhor desempenho dos alunos nas disciplinas mencionadas, após intervenção da abordagem Desplugada com as HQs.

\section{Introdução}

O Pensamento Computacional (PC) consiste na combinação do pensamento crítico e os conceitos de Ciência da Computação (CC) definido como uma abordagem para resolver problemas e auxiliando na estruturação do raciocínio. O PC pode ser aplicado na leitura, escrita e matemática, por exemplo, a fim de estimular habilidades analíticas [Wing 2006]. [Csizmadia et al. 2015] afirmam que o currículo da educação básica deve contemplar o desenvolvimento do PC. Recentemente, o PC foi inserido no contexto educacional brasileiro por meio da nova Base Nacional Comum Curricular (BNCC) como sendo uma das competências a ser desenvolvida na área de Matemática e suas Tecnologias, bem como o raciocínio lógico [Brasil 2017]. 
VIII Congresso Brasileiro de Informática na Educação (CBIE 2019)

Anais do XXV Workshop de Informática na Escola (WIE 2019)

Levando em consideração a situação socioeconômica brasileira, cerca de $50 \%$ das escolas públicas não possuem laboratórios de informática, e cerca de $60 \%$ dos alunos, $6^{\circ}$ ao $9^{\circ}$ ano, vivenciam uma aprendizagem insuficiente nas disciplinas de Língua Portuguesa e Matemática [MEC/INEP 2017] [IDEB 2018]. Dessa forma, [Brackmann 2017] sugere a computação "Desplugada" para o estímulo ao PC, que têm por objetivo ensinar os fundamentos sobre $\mathrm{CC}$ sem o uso de computadores, e/ou laboratórios de informática, sendo uma estratégia lúdica de estímulo ao PC na escola [Bell et al. 2007].

Dentre os artefatos Desplugados que podem ser usados para o estímulo do PC estão as Histórias em Quadrinhos (HQs) dos Almanaques para a Popularização de Ciência da Computação. De acordo com [Carvalho 2009], a utilização das HQs em sala de aula, estão atreladas a atração dos alunos por este tipo de leitura, que combinam imagens e linguagem simples, tornando uma forma eficiente de ensino, com qualidade em informações, proporcionando o enriquecimento da comunicação e do conhecimento, estimulando o hábito da leitura e do raciocínio lógico.

Considerando os aspectos acima mencionados, este trabalho propõe e relata a utilização de HQs com temáticas em CC, amparados pela abordagem Desplugada no estímulo do PC com estudantes do ensino fundamental, $9^{\circ}$ ano, nas disciplinas de Língua Portuguesa (interpretação textual) e Matemática (raciocínio lógico). O presente artigo está organizado da seguinte maneira: a seguir, na Seção 2 os Trabalhos Relacionados; na Seção 3 a Metodologia; Seção 4 Resultados e Discussão; Seção 5 Considerações Finais; e Referências.

\section{Trabalhos Relacionados}

No contexto do uso de atividades Desplugadas para o estímulo do PC, [Pires et al. 2018] investigaram se em narrativas infantis, na criação de histórias, emergem padrões correlacionados às variáveis consideradas essenciais para o desenvolvimento do $\mathrm{PC}$, com base nos processos de aprendizagem (Epistemologia Genética), a partir da neurociência cognitiva e da abstração, que os resultados preliminares apontam para a emergência de um padrão, mesmo nas histórias que podem ser classificadas como "sem roteiro", que se enquadre nas possibilidades de contar história pelo Scratch e, também, nos pilares do PC (decomposição, padrão, abstração e algoritmo). [Santos, Nunes and Romero 2019] apresentam os Pilares como estratégias para desenvolverem PC de forma Desplugada, utilizando recursos como HQs dos Almanaques para a Popularização de Ciência da Computação, papel e caneta. [Takahashi et al. 2009] relatam que o uso do livro "Guia Mangá de Banco de Dados", como um artefato Desplugado, auxiliou um grupo de jovens a entender os conceitos de Banco de Dados (BD) proporcionando a aprendizagem de forma acessível e lúdica.

[Silva and Cavalcanti 2018] avaliaram duas atividades Desplugadas, números binários e foguete a Marte, com 35 estudantes do curso técnico em informática, nível médio, do Instituto Federal de Pernambuco - Campus Garanhuns. Os resultados sugerem que as atividades apresentam potencial para estimular o $\mathrm{PC}$ dos alunos, que atingiram fluxo significativo (5,33 e 5,41 em uma escala de 0 a 7), com ( $p$-value $=0,93)$, atividade um, e (p-value $=0,74)$, atividade dois. [Souza and Lopes 2018] adaptaram atividades das Olimpíadas Brasileira de Informática (OBI) para estimular o PC de estudantes, por meio 
VIII Congresso Brasileiro de Informática na Educação (CBIE 2019)

Anais do XXV Workshop de Informática na Escola (WIE 2019)

da computação Desplugada, visando o desenvolvimento do raciocínio lógico é um processo fundamental para auxiliar na resolução de problemas em diversas áreas, inclusive na computação. Todavia, trabalhos relacionados, foram selecionados com a intenção de mensurar a influência do PC na aprendizagem na interdisciplinaridade e auxiliar na construção das atividades Desplugadas, sempre chegando à conclusão que o PC, de forma Desplugada, pode realmente aprimorar o raciocínio lógico e interpretação textual dos alunos.

\section{Metodologia}

A abordagem Desplugada foi aplicado com estudantes do ensino fundamental da Escola Municipal Antenor Serpa, na cidade de Olho d'Água do Casado-AL, entre os meses de setembro e outubro de 2018. Participaram 50 alunos que apresentavam faixa-etária entre 13 e 16 anos, $30 \%$ do gênero masculino e $70 \%$ do gênero feminino, $100 \%$ dos estudantes nunca tiveram contato com assuntos relacionados $\mathrm{CC}$ e não possuem computador em casa. Sendo realizadas atividades Desplugadas que ocorreram em sala de aula, como interventora nas disciplinas de Língua Portuguesa e Matemática, uma vez por semana durante 9 semanas, 50 minutos (cada) encontro.

Todavia, foram estudados durante os encontros, conceitos de lógica de programação, árvore binária, pilhas, filas e algoritmos, por meio da computação Desplugada, (utilizando caneta, papel, cola e tesoura) e HQs dos Almanaques para Popularização de Ciência da Computação, HQs, chanceladas pela Sociedade Brasileira de Computação (SBC), com o objetivo de estimular o PC dos estudantes nas disciplinas mencionadas anteriormente, acreditando na hipótese que as HQs junto a um conjunto de atividades Desplugadas interdisciplinares poderiam auxiliar no desenvolvimento do PC melhorando o rendimento escolar dos alunos. Assim, a execução da abordagem foi dividida em 4 etapas: (1) Exercício Inicial para Alunos (EIA), (2) Módulos de Ensino (ME), (3) Exercício Final para Alunos (EFA), e (4) Análise dos resultados.

As etapas (1) e (3) foram as aplicações dos exercícios, que contemplaram 54 questões (cada), adaptados [Yagci 2018], que buscam compreender a percepção dos alunos sob o conjunto de atividades, amparados por questões de (Resolução de Problemas; Aprendizado Cooperativo e Pensamento Crítico; Pensamento Criativo; e Pensamento Algorítmico). Todavia, para a mensuração dos exercícios, foi utilizada a escala Likert de 5 pontos invertida, (Discordo Totalmente, Discordo, Nem Concordo Nem Discordo, Concordo, Concordo Totalmente), que por meio do Alfa de Crombach, apresenta variação entre 0 e 1 , sendo possível averiguar a consistência das informações: superior a 0,9 - consistência muito boa; entre 0,8 e 0,9 - boa; entre 0,7 e 0,8 - razoável; entre 0,6 e 0,7 - fraca; inferior a 0,6 - inadmissível [Cronbach 1951]. Dessa forma, durante a mensuração da confiança dos exercícios os resultados devem apresentar valores iguais ou superiores a 0,7 . A etapa (2), consistiu na participação dos alunos nos Módulos de Ensino (ME) com atividades Desplugadas. Os ME foram divididos em 3: (ME1) Computação, Ética e Sociedade (4 encontros); (ME2) Estrutura de dados (3 encontros); e (ME3) Tempestade de Ideias (2 encontros).

A fase (4), deu-se início com a avaliação do rendimento escolar antes $\&$ após aplicação da abordagem Desplugada, comparando o $2^{\circ}$ bimestre (período em que os alunos não participaram dos encontros) e $3^{\circ}$ bimestre (período em que os alunos 
VIII Congresso Brasileiro de Informática na Educação (CBIE 2019)

Anais do XXV Workshop de Informática na Escola (WIE 2019)

participaram dos encontros). Dessa forma, foram utilizados testes de normalidade Kolmogorov-Smirnov e/ou Shapiro-Wilk; e Teste $t$ pareado, para comparar médias bimestrais antes \& após da participação dos alunos com as atividades Desplugadas, com o intuito de verificar se as médias dos alunos podem ser aproximada pela distribuição normal com base nas hipóteses nula (H0) ou alternativa (H1) [Pinheiro et al. 2015]. Assim, acreditando que um referente dado apresenta $\mathrm{H} 0$ quando a amostra provém de uma população normal $((\mathrm{p})>0,05)$; porém, diz-se que H1 quando a amostra não provém de uma população normal, assim o valor de $(p)$ será $((p)<0,05)$ [Shapiro et al. 2008]. Os testes de Normalidade devem apresentar grau de confiança de 95\% (nível de significância ( $\alpha$ ) de 5\%) [Pinheiro et al. 2015]. A realização do Teste t pareado visto que se trata da medição da mesma unidade amostral em momentos diferentes. Diz-se que H0 (distribuição da amostra igual a distribuição normal), ou seja, a amostra não apresenta diferença entre os valores $((p)>0,05)$; porém, diz-se que a H1 consiste em (distribuição da amostra quando diferencia da distribuição normal), sendo assim $((p)<0,05)$ [Pinheiro et al. 2015]. Todavia, para verificação estatística dos dados foi utilizando a ferramenta Statistical Package for the Social Sciences (SPSS).

\subsection{Descrição da Aplicação dos Módulos de Ensino}

No ME1 foram trabalhados os conceitos de PC, mulheres na Computação e profissão em Tecnologia da Informação (TI). Assim, para este ME foram utilizados os seguintes recursos: HQs ("Pensamento Computacional" (Série 7, Volume 1); "Conceitos Básicos sobre Programação e Scratch" (Série 1, Volume 7); "Computação, Jogos e Profissão" (Série 1, Volume 1); e "Mulheres na Computação" (Série 1, Volume 2)); papel A4; tesoura; cola para papel; papelão; e canetas hidrográficas. Todavia, no ME2, foram trabalhados os conceitos de Árvore Binária, Filas, Pilhas. Logo, foram utilizados os seguintes recursos: HQs (“Árvore Binária", (Série 5, Volume 2); "Pilhas", (Série 5, Volume 1); e "Filas", (Série 5, Volume 3)); papel A4; papelão; tesoura; e canetas hidrográficas. Já no ME3, foram trabalhados os conceitos apresentados durante o ME1 e ME2, incluindo os recursos. Na Tabela 1, apresenta as atividades e HQs por encontros semanais. Todas as HQs estão disponíveis de forma gratuita no site (http://almanaquesdacomputacao.com.br/).

Tabela 1. Encontros por Módulos de Ensino

\begin{tabular}{|c|l|l|l|}
\hline $\begin{array}{c}\text { Módulos } \\
\text { de Ensino }\end{array}$ & Encontros & \multicolumn{1}{|c|}{ HQs } & \multicolumn{1}{c|}{ Atividades } \\
\hline \multirow{3}{*}{ ME1 } & Encontro 1 & $\begin{array}{l}\text { HQ "Pensamento } \\
\text { Computacional” }\end{array}$ & $\begin{array}{l}\text { A hora do debate; Resolução de passatempos das HQs; } \\
\text { Destrinchando o Texto com Algoritmo Narrativo; } \\
\text { Destrinchando o Texto com Algoritmo Fluxograma; e } \\
\text { Dinâmica dos Movimentos das Estruturas Condicionais. }\end{array}$ \\
\cline { 2 - 5 } & Encontro 2 & $\begin{array}{l}\text { HQ "Conceitos } \\
\text { Básicos sobre } \\
\text { Programação e } \\
\text { Scratch" }\end{array}$ & $\begin{array}{l}\text { A hora do debate; Resolução de passatempos das HQs; } \\
\text { Destrinchando o Texto com Algoritmo Narrativo; } \\
\text { Destrinchando o Texto com Algoritmo Fluxograma; e } \\
\text { Quebra-cabeça Desplugado com Scratch. }\end{array}$ \\
\cline { 2 - 4 } & Encontro 3 "Computação, & $\begin{array}{l}\text { HQ "Cora do debate; Resolução de passatempos das HQs; } \\
\text { Jogos e Profissão" } \\
\text { e Destrinchando o Texto com Algoritmo Narrativo. }\end{array}$ \\
\cline { 2 - 4 } & Encontro 4 & $\begin{array}{l}\text { HQ "Mulheres na } \\
\text { Computação" }\end{array}$ & $\begin{array}{l}\text { A hora do debate; Resolução de passatempos das HQs; } \\
\text { e Destrinchando o Texto com Algoritmo Narrativo. }\end{array}$ \\
\hline
\end{tabular}


VIII Congresso Brasileiro de Informática na Educação (CBIE 2019)

Anais do XXV Workshop de Informática na Escola (WIE 2019)

\begin{tabular}{|c|l|l|l|}
\hline \multirow{2}{*}{ ME2 } & Encontro 5 & $\begin{array}{l}\text { HQ “Árvore } \\
\text { Binária" }\end{array}$ & $\begin{array}{l}\text { A hora do debate; Resolução de passatempos das HQs; } \\
\text { Destrinchando o Texto com Algoritmo Narrativo; e } \\
\text { Desafio de Raciocínio - Lógico Árvore Binária e } \\
\text { Genealógica. }\end{array}$ \\
\cline { 2 - 4 } & Encontro 6 & HQ "Filas" & $\begin{array}{l}\text { A hora do debate; Resolução de passatempos das HQs; } \\
\text { Destrinchando o Texto com Algoritmo Narrativo; } \\
\text { Dinâmica do pense rápido antecessor e sucessor; e } \\
\text { Desafio de Raciocínio Lógico - Organizando a Fila. }\end{array}$ \\
\cline { 2 - 5 } & Encontro 7 & HQ "Pilhas" & $\begin{array}{l}\text { A hora do debate; Resolução de passatempos das HQs; } \\
\text { Destrinchando o Texto com Algoritmo Narrativo; e } \\
\text { Desafio de Raciocínio Lógico - 20 Pilhas. }\end{array}$ \\
\hline ME3 & Encontro 8 & $\begin{array}{l}\text { Todas as HQs } \\
\text { utilizadas neste } \\
\text { experimento. }\end{array}$ & $\begin{array}{l}\text { Desafio de Raciocínio Lógico - Elaborando Desafio. } \\
\text { Criando Histórias. }\end{array}$ \\
\cline { 2 - 4 } & Encontro 9 & \\
\hline
\end{tabular}

Dessa forma, para cada encontro semanal, uma HQ era utilizada e consigo atividades Desplugadas, que foram desenvolvidas com base nos trabalhos relacionados, dentro da temática abordada.

\subsection{Descrição das atividades dos Módulos de Ensino}

$\mathrm{Na}$ atividade "A hora do debate", os alunos foram estimulados por meio de questionamentos sobre o conteúdo estudado a relacionar as temáticas abordadas ao cotidiano realizando analogias; "Passatempos ao final das HQs" ao final de cada HQ contempla atividades relacionadas ao conteúdo estudado, assim, os alunos foram submetidos a resolverem os passatempos com o intuito de fixar o assunto apresentado no encontro (aula); Na atividade "Destrinchando o texto com Algoritmo Narrativo" os alunos organizavam o entendimento a respeito da leitura, seguindo a estrutura lógica de um Algoritmo Narrativo escrevendo em um papel A4. Exemplo: Passo 1: "escrever o título da obra", Passo 2: "escrever a temática abordada", Passo 3: "realizar analogias "e assim sucessivamente.

A atividade "Destrinchando o texto com Algoritmo Fluxograma" tinha como objetivo, que os alunos estruturassem o entendimento referente a leitura realizada na HQ, seguindo uma estrutura lógica elaborando um Algoritmo Fluxograma em um papel A4 utilizando canetas hidrográficas coloridas para desenhar as formas geométricas (símbolos), e no interior do desenho escrever o que cada forma solicitava, conforme Tabela 2. A atividade "Quebra-cabeça com Scratch Desplugado" são blocos de comandos impressos, colados no papelão e recortados, do site oficial da ferramenta Scratch, que os alunos recebiam desafios de raciocínio lógico e tinha que resolver o quebra-cabeça com os blocos Desplugados no menor tempo possível. Já na "Dinâmica dos Movimentos das Estruturas Condicionais" eram verbalizadas ações corporais relacionadas a alguma das estruturas condicionais, (SE; SENÃO), e os alunos reproduziam ação. À vista disso, ganha a atividade aquele que chegar ao final sem errar nenhuma instrução. Exemplo 1: "SE eu bater as mãos, então vocês batem os pés"; Exemplo 2: "SE eu NÃO bater os pés, vocês podem pular".

Tabela 2. Símbolos para Construção de Fluxogramas

\begin{tabular}{|l|l|l|l|l|l|l|}
\hline Símbolo & & & & & & \\
\hline Nome & Início/ Fim & Decisão & Processamento & Documento & Informações & Fluxo de \\
\hline
\end{tabular}


VIII Congresso Brasileiro de Informática na Educação (CBIE 2019)

Anais do XXV Workshop de Informática na Escola (WIE 2019)

\begin{tabular}{|l|l|l|l|l|l|l|}
\hline & & & & & & linha \\
\hline $\begin{array}{l}\text { Quando } \\
\text { utilizar }\end{array}$ & $\begin{array}{l}\text { Iniciar e } \\
\text { finalizar o } \\
\text { fluxograma }\end{array}$ & $\begin{array}{l}\text { Escrever } \\
\text { Verbos }\end{array}$ & $\begin{array}{l}\text { Escrever } \\
\text { Substantivos e } \\
\text { Adjetivos }\end{array}$ & $\begin{array}{l}\text { Temática do } \\
\text { texto }\end{array}$ & $\begin{array}{l}\text { Resumo do } \\
\text { texto }\end{array}$ & $\begin{array}{l}\text { Direção do } \\
\text { fluxograma }\end{array}$ \\
\hline
\end{tabular}

No desafio de raciocínio lógico “Árvore Binária e Genealógica” cada aluno retirou de uma sacola 10 tipos de parentescos, em seguida os alunos montaram, individualmente, uma árvore genealógica com os parentescos sorteados, vale ressaltar que podem ser sorteados parentescos iguais. Outro desafio, "Organizando a Fila" cada aluno recebeu um questionário elaborados pelos professores de Língua Portuguesa e Matemática, com 10 problemas matemáticos relacionados aos conteúdos trabalhados em sala de aula, após responderem todas as questões os alunos organizaram as repostas em uma fila na ordem crescente. O desafio "20 Pilhas" os alunos formaram duplas e elaboraram 20 diferentes tipos de analogias relacionadas ao cotidiano baseadas no conceito de Pilhas, que tinham tempo máximo de 10 minutos para finalizar o desafio. A dupla vencedora foi a que apresentar a menor quantidade de repetições de analogias quando comparadas com as outras duplas.

Na atividade "Dinâmica do Pense Rápido Antecessor/Sucessor", cada aluno elaborou 10 cards com problemas matemáticos envolvendo raciocínio lógico e interpretação textual. Em seguida, formaram duplas e cada aluno desafiou o outro, para cada desafio o adversário tinha 1 minutos para resolver, os vencedores de cada dupla participaram de uma rodada final com um único card elaborado pelos professores de Língua Portuguesa e Matemática, ganhou o aluno que respondeu em menor tempo. Exemplo 1: jogador 1 pergunta para jogador 2 "O antecessor da raiz quadrada de 25?"; Exemplo 2: "Existe uma fila com 3 pessoas João, Marcos e André. Marcos é o primeiro da fila, André é o terceiro da fila, quem é o sucessor de Marcos?". O "Elaborando Desafios", nesta atividade foram formados 7 grupos, que cada grupo ficou responsável por uma HQ estudada anteriormente, em seguida, os componentes de cada grupo elaboraram um desafio para os outros grupos que foram solucionados por meio da atividade "Destrinchando o Texto com Algoritmo Narrativo", consequentemente, o grupo que terminou todos os desafios primeiro foi o vencedor. Por fim, "Criando Histórias" que cada aluno elaborou uma história em quadrinho utilizando 9 quadrinhos, em um papel A4, e apresentou para os outros alunos, essas histórias apresentavam analogias ao cotidiano sobre os conceitos estudados. Sendo assim, após aplicação dos ME e EFA, deu-se início a análise e discussão dos resultados.

\section{Resultados e Discussão}

Inicialmente, os exercícios passaram por uma análise de consistência das respostas meio do Alfa de Crombach, ambos os exercícios EIA e EFA apresentaram consistência (razoável), já que dispõem do valor igual ou superior a $(0,7)$. Logo, o EIA apresenta consistência $(0,7)$ para $(\mathrm{N}=50)$ e (Itens $=54)$, e o EFA apresenta consistência $(0,728)$ para $(\mathrm{N}=50)$ e (Itens $=54)$, conforme Tabela 3. A análise detalhada e questões dos exercícios, estão disponíveis no link: https://drive.google.com/drive/folders/1rsrur4tZkLKG19WRqH0OQZ2xOMWuqVjS?u $\underline{\mathrm{sp}}=$ sharing .

Tabela 3. Resultados dos exercícios

\begin{tabular}{|l|l|l|l|l|}
\hline Exercícios & $\mathrm{N}$ (Participantes) & ITENS (Questões) & Alfa de Crombach & Consistência \\
\hline
\end{tabular}


VIII Congresso Brasileiro de Informática na Educação (CBIE 2019)

Anais do XXV Workshop de Informática na Escola (WIE 2019)

\begin{tabular}{|l|l|l|l|l|}
\hline EIA & 50 & 54 & 0,700 & Razoável \\
\hline EFA & 50 & 54 & 0,728 & Razoável \\
\hline
\end{tabular}

Dessa forma, os resultados apresentados nos EIA e EFA caracterizam-se (confiabilidade do teste-reteste), pois, a consistência dos resultados identificados foi analisada em momentos, antes \& após aplicação da abordagem, diferentes pelo mesmo instrumento de medição, o EIA e EFA [Landis and Koch 1977]. Entretanto, é identificado no EIA que os alunos tinham dificuldades em interpretação textual e raciocínio lógico, pois, os alunos foram questionados se tinham dificuldades ou não em raciocínio lógico, interpretação textual, criatividade, proatividade, pensamento sequencial, planejamento de tarefas, solução de problemas, aprendizagem cooperativa e pensamento algoritmo. Todavia, depois da aplicação dos ME em resposta ao EFA, os alunos passaram a concordar que as HQs e os conceitos de CC podem auxiliar no processo de ensino-aprendizagem no desenvolvimento do raciocínio lógico, interpretação textual, PC, pensamento crítico, pensamento algoritmo, pensamento cooperativo, e na resolução de problemas. Dessa forma, os resultados apresentados podem sofrer ameaças à validade, assim, interferindo na mensuração dos resultados. Depois da análise dos exercícios, deu-se início a análise do rendimento antes $\&$ após aplicação da abordagem Desplugada. Dessa forma, foram realizados os testes de normalidade das médias bimestrais, conforme Tabela 4 .

Tabela 4. Confiabilidade testes de normalidade

\begin{tabular}{|c|c|c|}
\hline Bimestre & Kolmogorov-Smirnov & Shapiro-Wilk \\
\hline $2^{\circ}$ Língua Portuguesa & 0,091 & 0,208 \\
\hline $3^{\circ}$ Língua Portuguesa & 0,084 & 0,172 \\
\hline $2^{\circ}$ Matemática & 0,064 & 0,163 \\
\hline $3^{\circ}$ Matemática & 0,700 & 0,062 \\
\hline
\end{tabular}

Conforme apresentado na Tabela 4, no $2^{\circ}$ bimestre em Língua Portuguesa, obteve-se para Kolmogorov-Smirnov (p-value $=0,091)$ e Shapiro-Wilk (p-value $=$ 0,208). Já, os valores de (p) referentes ao $3^{\circ}$ bimestre para Kolmogorov-Smirnov (pvalue $=0,084)$ e Shapiro-Wilk ( $\mathrm{p}$-value $=0,172)$. Na disciplina de Matemática, atingiuse em Kolmogorov-Smirnov (p-value $=0,064)$ e Shapiro-Wilk (p-value $=0,163)$, já os valores de (p) referentes ao $3^{\circ}$ bimestre foram: Kolmogorov-Smirnov ( $\mathrm{p}$-value $\left.=0,070\right)$ e Shapiro-Wilk (p-value $=0,062)$. Desse modo, ambos os testes de normalidade apresentaram em seus resultados para $(\mathrm{p})$ valores superiores a $(0,05)$, assim, acredita-se que os valores apresentados consistem em ser considerados dados normais amparados pela hipótese nula (H0), quando a amostra provém de uma população normal, assim, $((p)>0,05)$. Nesse ínterim, depois da realização dos testes de normalidade, deu-se início à análise referente ao Teste $t$ pareado, que foram identificados os seguintes valores para Média (M) e Erro Padrão (EP), conforme apresentado na Tabela 5.

Tabela 5. Teste t pareado

\begin{tabular}{|l|l|l|l|l|l|}
\hline Bimestre & $\mathrm{M}$ & $\mathrm{EP}$ & Grau de Liberdade & Valor Grau de Liberdade & $\mathrm{P}$ \\
\hline $2^{\circ}$ Língua Portuguesa & 4,714 & 0,2320 & 50 & 13,787 & 0,000 \\
\hline $3^{\circ}$ Língua Portuguesa & 7,884 & 0,1521 & 50 & 13,787 & 0,000 \\
\hline $2^{\circ}$ Matemática & 4,654 & 0,2329 & 50 & 13,609 & 0,000 \\
\hline
\end{tabular}


VIII Congresso Brasileiro de Informática na Educação (CBIE 2019)

Anais do XXV Workshop de Informática na Escola (WIE 2019)

$3^{\circ}$ Matemática 7,868

0,1589

50

13,609

0,000

Dessa forma, conforme apresentado na Tabela 5, a turma obteve em Língua Portuguesa no $2^{\circ}$ bimestre $(\mathrm{M}=4,714)$ com $(\mathrm{EP}=0,2320)$. Já no $3^{\circ}$ bimestre $(\mathrm{M}=7,884)$ com $(\mathrm{EP}=0,1521)$. Desse modo, o valor de $(t)$ é $(\mathbf{t}(\mathbf{5 0})=\mathbf{1 3 , 7 8 7},(\mathbf{p}<\mathbf{0 , 0 5}))$, assim, $(t)$ apresenta o grau de liberdade $(50)$ com valor correspondente a $(13,787)$ e $(\mathrm{p}$-value $=$ 0,000). Todavia, $2^{\circ}$ bimestre a turma em Matemática obteve $(\mathrm{M}=4,654)$ com $(\mathrm{EP}=0,2329)$, e no $3^{\circ}$ bimestre $(\mathrm{M}=7,868)$ com $(\mathrm{EP}=0,1589)$. Portanto, o valor de $(t)$ é (t $(50)=13,609,(\mathbf{p}<\mathbf{0 , 0 5}))$, logo $(t)$ possui o grau de liberdade $(50)$ com valor correspondente a $(13,609)$ e $(p$-value $=0,000)$, conforme apresentado na Tabela 5 . Dessa maneira, diz-se que o valor de $(t)$ coincide com a hipótese alternativa (H1) quando a distribuição da amostra diferencia da distribuição normal, $\operatorname{logo},((\mathrm{p})<0,05)$. À vista disso, certifica-se que as HQs junto a um conjunto de atividades Desplugadas podem auxiliar no desenvolvimento do $\mathrm{PC}$, visto que a turma obteve um acréscimo de $(+3,17)$ na média da disciplina de Língua Portuguesa e acréscimo de $(+\mathbf{3}, 214)$ na disciplina de Matemática, após utilização das HQs no $3^{\circ}$ bimestre. Todavia, o Teste $t$ pareado certificou que as HQs dos Almanaques para Popularização de Ciência da Computação contribuíram para o avanço na aprendizagem dos alunos nas disciplinas de língua Portuguesa e Matemática, em uma análise com 95\% de intervalo de confiança. No entanto, os resultados apresentados podem sofrer ameaças à validade, assim, interferindo na mensuração dos resultados.

\subsection{Ameaças à Validade}

Diferentes problemas podem ser ocasionados durante a participação dos indivíduos no experimento antes \& após aplicação da abordagem Desplugada. Instrumentação adequada preparada, (validade interna): os participantes responderam aos exercícios sem nenhuma supervisão, assim, os mesmos podem não terem entendido alguma questão específica; A quantidade de questões pode interferir no valor apresentado pelo Alfa de Crombach, visto que questionários longos podem aumentam o valor de alfa, e questionários curtos podem diminuir o valor de alfa, sem que isso signifique aumento de consistência interna [Landis and Koch 1977].

População representativa, (validade externa): o número de participantes para responderem aos questionários e analisar rendimento, antes \& depois aplicação da abordagem Desplugada podem ser uma ameaça, sendo capaz de influenciar nos resultados para validação. Outro fator é que toda a turma participou, assim, inviabilizando a possibilidade de comparação de rendimento entre 2 grupos de controle de uma mesma sala. Distribuição do conjunto de participantes (validade de conclusão): os exercícios EIA e EFA foram aplicados antes \& após aplicação da abordagem Desplugada com as mesmas questões.

\section{Considerações Finais}

Com o desenvolvimento das atividades Desplugadas, percebe-se, na prática, o quanto o uso das HQs dos Almanaques para Popularização de Ciência da Computação tem se mostrado uma estratégia de ensino promissora para o desenvolvimento do PC. As atividades Desplugadas apresentadas com base nas HQs, estão apresentando os seus primeiros resultados coletados, avaliados e discutidos. À vista disso, a escola participante juntamente 
VIII Congresso Brasileiro de Informática na Educação (CBIE 2019)

Anais do XXV Workshop de Informática na Escola (WIE 2019)

com professores envolvidos, alunos atendidos e equipe pedagógica da escola que acompanhou a execução das atividades desenvolvidas, apoiam as atividades Desplugadas, já que as mesmas auxiliaram no processo de ensino- aprendizagem nas disciplinas de Língua Portuguesa (interpretação textual) e Matemática (raciocínio lógico). O aumento gradativo das notas dos alunos é notório, a turma obteve um acréscimo de $(+3,17)$ na média da disciplina de Língua Portuguesa e acréscimo de $(+3,214)$ na disciplina de Matemática após utilização das HQs, atingindo o objetivo e validando a hipótese que as HQs associadas a um conjunto de atividades Desplugadas interdisciplinares, auxiliam no desenvolvimento do PC melhorando o rendimento escolar dos alunos. Como trabalhos futuros, pretende-se ampliar para as outras disciplinas da grade curricular dos alunos do ensino fundamental, para diminuir as ameaças à validade, almeja-se diminuir a quantidade de questões e supervisionar a resolução dos exercícios.

\section{Referências}

Bell, T., Curzon, P., Cutts, Q., Dagiene, V., and Haberman, B. (2011). Overcoming obstacles to cs education by using non-programming outreach programmes. In International Conference on Informatics in Schools: Situation, Evolution, and Perspectives, pages $71-81$. Springer.

Blikstein, P. (2008). O pensamento computacional e a reinvenção do computador na educação. Disponível em $<$ http://www.blikstein.com/paulo/documents/online/ol_pensamento_computacional.html $>$, Acessado em: 10 Jul 2017

BRASIL. Ministério da Educação. Secretaria da Educação Básica. Base nacional comum curricular. Brasília, DF, 2017. Disponível em: < http://basenacionalcomum. mec.gov.br >. Acesso em: abr.2018

Brackmann, C. P., Roman-Gonzalez, M., Robles, G., Moreno-Leon, J., Casali, A., and Barone, D. (2017b). Development of computational thinking skills through unplugged activities in primary school. In Proceedings of the 12th Workshop on Primary and Secondary Computing Education, pages 65-72. ACM.

Carvalho, J.. (2009). Trabalhando com quadrinhos em sala de aula. CECIERJ - Educação Pública. Disponível em: http://www.educacaopublica.rj.gov.br/suavoz/0116.html> Acessado em: 10 Jul 2017

Cronbach, L J. Coefficient Alpha and the internal structure of tests. Psychometrika 16:297334, 1951.

Gadotti, M. Perspectivas atuais da educação. Porto Alegre, Ed. Artes Médicas, 2000.

Csizmadia, A.; Curzon, P.; Dorling, M.; Humphreys, S.; Thomas Ng; Selby, C.; Woollard, J.. (2015). Computational thinking: a guide for teachers. Computing At School. Disponível em: <http://community.computingatschool.org.uk/files/6695/origin al.pdf $>$.

Guarda, G. F. and Goulart, I. F. (2018) "Jogos Lúdicos sob a ótica do Pensamento Computacional: Experiências do Projeto Logicamente" VII Congresso Brasileiro de Informática na Educação (CBIE 2018). Anais do XXIX Simpósio Brasileiro de Informática na Educação (SBIE 2018).

IDEB. Resultados E Metas (2018). Disponível em: $<$ http://ideb.inep.gov.br/resultado/resultado/resultadoBrasil.seam?cid=4353105> Acessado em: 20 Set 2018 
VIII Congresso Brasileiro de Informática na Educação (CBIE 2019)

Anais do XXV Workshop de Informática na Escola (WIE 2019)

Landis, J. R., and Koch, G. G. (1977) The measurement of observer agreement for categorical data. Biometrics. 33:159.

Machado, E. Z. A., Vasconcelos, I. R., Amorim, K. M., Andrade, A. M. S.; Barreto, L. P. and Santos, D. A. . "Uma Experiência em Escolas de Ensino Médio e Fundamental para a Descoberta de Jovens Talento sem Computação". In: XVIII Workshop sobre Educação em Computação, 2010, Belo Horizonte. Anais do XXX CSBC, 2010.

MEC/INEP. Anuário Brasileiro da Educação Básica / Todos pela Educação., (2017). Editora Moderna. Disponível em: $<$ https://www.todospelaeducacao.org.br/biblioteca/1545/anuario-brasileiro-daeducacaobasica-2016/>.

Pinheiro, J. I. D.; Cunha, S. B.; Carvajal, S. R.; Gomes, G. C. Estatística Básica: A arte de trabalhar com dados. $2^{\mathrm{a}}$ edição - Rio de Janeiro: Editora Campus ELSEVIER (2015). 360 .

Pires, F., Duarte, J. C., Pessoa, L., Pereira, K. S., Ferreira, R. and Freitas, R. (2018). "Uma análise cognitiva entre a emergência de padrões em narrativas infantis e elementos do Pensamento Computacional". VII Congresso Brasileiro de Informática na Educação (CBIE 2018). Anais do XXIX Simpósio Brasileiro de Informática na Educação (SBIE 2018).

Santos, C. G. (2019) Estratégias Para Implantação E Avaliação De Um Método Educacional Desplugado Com Histórias Em Quadrinhos Para O Ensino E Aprendizagem Associados Ao Desenvolvimento Do Pensamento Computacional Com Alunos Do Ensino Fundamental.. 2019. Dissertação de mestrado (PROCC) - (UFS) Universidade de Sergipe.

Silva, L. S. and Cavalcante, E. R. (2018)."Avaliac, ao do Estado de Fluxo e do Aprendizado em Atividades Desplugadas no Ensino do Pensamento Computacional com Estudantes do Ensino Medio". VII Congresso Brasileiro de Informática na Educação (CBIE 2018). Anais do XXIX Simpósio Brasileiro de Informática na Educação (SBIE 2018).

Shapiro, S. L., Oman, D., Thoresen, C. E., Plante, T. G., and Flinders, T. (2008). Cultivating Mindfulness : Effects on Well-Being. 64(7):840-862.

Souza, J. S. and Lopes, A. S. B. (2018). "Estimulando o pensamento computacional e o raciocínio lógico no ensino fundamental por meio da OBI e computação desplugada". VII Congresso Brasileiro de Informática na Educação (CBIE 2018). Anais do XXIX Simpósio Brasileiro de Informática na Educação (SBIE 2018).

Takahashi, M.; Azuma, S.; trend-pro co., LTD. Guia Mangá de Banco de Dados. 1o ed. Tokyo, Japão: Novatec, 2009.

Wing, J. M. Computational Thinking: What and Why? (2010). Disponível em: $<$ http://www.cs.cmu.edu/ CompThink/resources/TheLinkWing.pdf $>$. Acesso em: 23 Nov 2015.

Yagci, M. (2018) A valid and reliable tool for examining computational thinking skills. Disponível em: $\quad<$ https://link-springercom.ez20.periodicos.capes.gov.br/article/10.1007/s10639-018-9801-8>.Acessado em: 20 Set 2018 